\title{
gु \\ Addition spectra of Wigner islands of electrons on superfluid helium
}

\author{
Emmanuel Rousseau, ${ }^{*}$ Dmitri Ponarin, ${ }^{\dagger}$ Likourgos Hristakos, ${ }^{\ddagger}$ Olivier Avenel, Eric Varoquaux, and Yuri Mukharsky \\ Service de Physique de l'État Condensé Centre de Saclay, 91191 Gif-sur-Yvette cedex, France
}

(Received 8 August 2008; published 12 January 2009)

\begin{abstract}
We present here an experimental study of Wigner islands formed by electrons floating over helium. Electrons are trapped electrostatically in a mesoscopic structure covered with a helium film, behaving as a quantum dot in the near-classical limit. By removing electrons one by one, we are able to find the addition spectrum, i.e., the energy required to add (or extract) one electron from the trap with occupation number $N$. Experimental addition spectra are compared with Monte Carlo simulations for the actual trap geometry, confirming the ordered state of electrons over helium in the island.
\end{abstract}

DOI: 10.1103/PhysRevB.79.045406

PACS number(s): 74.78.Na, 73.21.Fg

\section{INTRODUCTION}

Electrons collect on a two-dimensional (2D) sheet when they are spread over the surface of a liquid-helium film. This is due to the attractive charge which appears by polarization of the helium. Due to the weakness of the image charge, electrons sit in the vacuum far away from the surface and move freely at a fixed distance over the film. As such, they constitute a very clean and predictable system.

When temperature is reduced, kinetic energy decreases relative to Coulomb energy; correlations begin to dominate the electronic structure. Wigner ${ }^{1}$ predicted in 1934 that a phase transition would take place in the infinitely extended system, leading to the formation of a 2D electron lattice. Wigner crystallization into a triangular lattice of electrons over helium has been observed first by Grimes and Adams ${ }^{2}$ $\left(\right.$ see also Shikin $^{3}$ ). This phase transition takes place below a transition temperature that is a function of electronic density. For the infinite system in the classical limit, ${ }^{4}$ in which particles can be treated individually and which is mostly appropriate for electrons over helium, the transition occurs when the ratio $\Gamma$ of the average Coulomb interaction energy $E_{C}$ to the thermal energy $k_{B} T$ becomes greater than 137 . In the quantum case, which is eventually reached at low enough temperatures, the Wigner solid is predicted to form when the Brueckner parameter $r_{s}$, which is the interelectron distance normalized by the Bohr radius, $a_{B}$, becomes smaller than 37 . Recently, somewhat more complex phases and ordering transitions have been predicted around that $r_{s}$ threshold. 5,6

An assembly of rectilinear vortices provides another example of Wigner lattice formation.,8 Abrikosov's triangular lattice for vortices induced by magnetic fields in type II superconductors $^{9}$ was first observed indirectly by neutron diffraction ${ }^{10}$ and later visualized directly by electron microscopy imaging by Bitter decoration of the trapped flux lines with ferromagnetic microparticles. ${ }^{11}$

Also, electrons in semiconductor heterostructures localized by an applied magnetic field undergo a magnetically induced Wigner transition. ${ }^{12}$ They form a well-studied and well-understood system: the quantum dot, which has been the object of extensive research for the past two decades. ${ }^{13}$

\section{A. Wigner molecules}

When confining 2D electrons to a restricted planar area, the breaking of translation invariance brings in important changes with respect to the infinite geometry case, in particular because thermodynamic phase transitions are suppressed by fluctuations. This problem of $2 \mathrm{D}$ ordering in restricted geometries has been extensively studied theoretically, mostly for parabolic traps with Coulomb interaction between the electrons: first in the limit where electrons behave as classical particles, ${ }^{8,14-16}$ and then, in the computationally more demanding quantum case. ${ }^{17-20}$

Finite-size effects become prevalent for $N \lesssim 100$. Such is the case in the work described below. Different features appear that depend on the competition between the triangular lattice, which takes over for sufficiently large systems, and the shape and strength of the confining potential, which tends to suppress it. The electron states and energies depend markedly on the trap geometry and on the details of the interparticle interaction, which in turn depends on the number of electrons in the trap and their arrangement.

For traps with hard confining walls and a flat trap bottom, it is predicted that the ordering is mostly affected close to the boundaries. Electrons in the interior of the $2 \mathrm{D}$ island tend to retain the triangular lattice structure and adjust to the walls at the trap periphery with a disorganized layer. For traps with cylindrical symmetry and a parabolic confining potential, the particles arrange themselves in circular concentric shells with widths that are small compared to the radius of the shell. These structures follow a Hund-type law ${ }^{14}$ and are referred to as Wigner molecules. The direct observation of such structures in restricted geometry has been achieved only for systems of macroscopic charged particles, ${ }^{21}$ and for vortices in superconductors ${ }^{22}$ and superfluid helium. ${ }^{23,24}$

For electrons over helium, finite islands of electrons can now be realized ${ }^{25}$ so that well-controlled experiments can be conducted on this very clean system, which we report here. Direct (visual) observation of the electronic structure cannot be performed in the present experiments: the geometric arrangement of the electrons in the island must be deduced from properties such as their escape energy from the island.

Contrarily to solid-state quantum dots, for which interactions between electrons decrease exponentially due to the screening from surrounding electrodes, electrons over helium are located far from conducting bodies; the Coulomb interaction is mostly unscreened and gives rise to strong, longrange, interparticle correlations. Also, there are no nearby impurities and no effective-mass correction; image charges 
are well defined. These features concur to make Wigner islands of electrons over helium an ideal model for the study of strongly correlated few-body fermionic systems and the formation of Wigner molecules.

\section{B. "Phase" diagram}

Various ordering processes take place in 2D clusters of electrons - the Wigner islands - and the formation of radially correlated structures - the Wigner molecules-have been the subject of a large number of theoretical studies. Our interest here lies in systems with a small number of electrons, $N$ $\lesssim 20$, confined in relatively large traps so that the electronic density is low.

The behavior of $N$ electrons freely suspended in vacuum over liquid helium and confined laterally in a circular parabolic trap by a potential $\frac{1}{2} m_{e} \omega_{0}^{2} r^{2}, m_{e}$ being the bare electron mass and $\omega_{0} / 2 \pi$ being the harmonic trap frequency, is described by the following Hamiltonian:

$$
\mathcal{H}=\sum_{i=1}^{N}\left\{\frac{\hbar^{2} \nabla_{i}^{2}}{2 m_{e}}+\frac{m_{e} \omega_{0}^{2}}{2} r_{i}^{2}\right\}+\sum_{i<j}^{N} \frac{e^{2}}{4 \pi \epsilon_{0}\left|\mathbf{r}_{i}-\mathbf{r}_{j}\right|} .
$$

The terms in curly brackets describe noninteracting electrons in the trap. The characteristic length $l_{0}=\left(\hbar / m_{e} \omega_{0}\right)^{1 / 2}$ associated with these two terms can be viewed as the spatial extent of electronic motion. The Coulomb interaction, which is the last term in Eq. (1) in which $e$ is the electron charge, eventually localizes and orders the electrons within the trap to distances of the order of $r_{0}$ such that $E_{C}=e^{2} / 4 \pi \epsilon_{0} r_{0}$ $=\frac{1}{2} m_{e} \omega_{0}^{2} r_{0}^{2}$. Following Filinov et al., ${ }^{18}$ we can therefore take the quantity $\bar{n}=\sqrt{2} l_{0}^{2} / r_{0}^{2}$, which represents the fraction of the trap area actually occupied by the electrons as a dimensionless measure of the electronic areal density.

The configuration and energies of Wigner islands with $N$ electrons are described by Eq. (1), the eigenstates of which however can be found analytically only for $N \leq 2 .{ }^{26}$ Various approximation schemes have to be used for few-body occupation number. ${ }^{27-29}$ Larger electron islands require a numerical approach (see, for a review, Reimann and Manninen ${ }^{20}$ ). Interactions with the environment-heat bath and measuring equipment-are not included in Eq. (1). In the simulations of Filinov et $a l .{ }^{18}$ the temperature comes in as an input in the Monte Carlo (MC) simulations.

A generalized "phase" diagram for the various ordering processes taking place in Wigner islands with few electrons has been constructed numerically by Filinov et al. ${ }^{18}$ whose findings are sketched in Fig. 1. These authors characterize the onset of order by looking at the correlations between electrons both radially and angularwise. In the ordered state, they find that $r_{0}$, introduced above on dimensional grounds, is quite close to the mean interparticle distance defined by the first maximum of the pair distribution function. When coming from the dense, hot (large $\bar{n}$ and/or $T$ ) regions of the diagram, where the 2D electron cluster is in a disordered (liquid or gaslike) state and moving to more dilute, cooler region, boundary lines are found first to the radially ordered state [radially "melting" (RM)], and, moving further in, to complete ordering where the orientation of the various shells

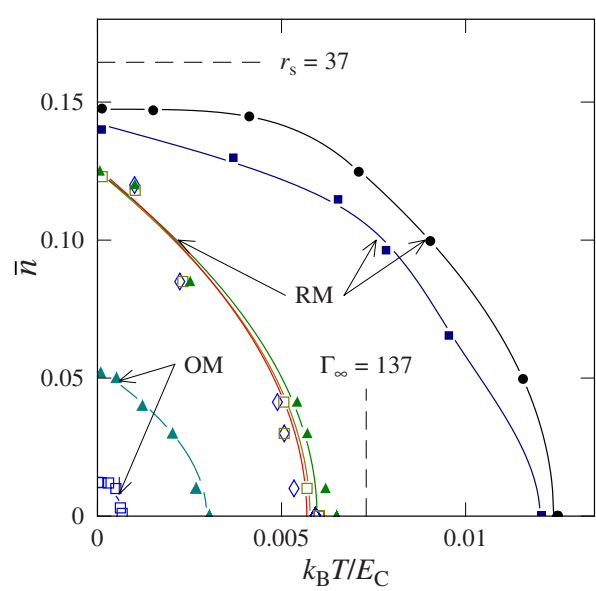

FIG. 1. (Color online) RM and OM "melting" in Wigner islands sketched in the $\bar{n}$ vs $k_{B} T / E_{C}$ plane for small electron occupation numbers $N-(\diamond) 10-(\bullet) 11-(\square) 12-(\boldsymbol{\Delta}) 19-(\boldsymbol{\square})$ 20, following Filinov et al. (Ref. 18). The OM boundaries for $N=10$, 11, and 20 are very close to the origin and are not shown. The horizontal (vertical) dash-dash lines indicate the quantum (classical) Wigner crystallization in the infinite system, for $r_{s}=1 / n^{2}=37$ and $\Gamma_{\infty}$ $=E_{C} / k_{B} T=137$, respectively.

become frozen with respect to one another [orientational melting $(\mathrm{OM})]$.

\section{Electronic configurations}

As seen in Fig. 1, Wigner ordering takes place at low densities $\bar{n}$ and low reduced temperatures $k_{B} T / E_{C}$ along boundaries that differ markedly for various occupation numbers $N$. Some clusters are more stable than others. Specifically, clusters with $N=11$ and 20 in Fig. 1 melt radially at higher $T, \bar{n}$ but orientationally at much lower values of $T, \bar{n} .{ }^{18}$

The orientationally more stable clusters, the magic clusters, $N=10,12,16$, and 19 in Ref. 18 , possess a fully frozen structure that is closest to the Wigner triangular lattice so that the shells cannot rotate on one another. Those that can, $N$ $=11$ and 20, are far less stable orientationally but a little more stable radially.

Somewhat surprisingly, these more radially stable configurations turn out to be even more stable than the homogeneous Wigner crystal itself, which melts in the classical limit for temperatures above that set by $\Gamma_{\infty}=137$. For the full quantum case, the critical melting density for the homogeneous system is set by the value of the Brueckner parameter $r_{s} \equiv r_{0} / a_{B}=37$. The Brueckner parameter can be extended to finite systems by taking the definition of $r_{0}$ in an electron cluster given above, which yields $r_{s}=1 / \bar{n}^{2}$. All boundary lines in Fig. 1 lie below the melting curve for the quantum Wigner crystal. ${ }^{30}$

These boundaries do not correspond to sharp transitions between electronic structures with different types of order. The localization process of the electrons into organized structures takes place only gradually, as stressed by Ghosal et $a l .{ }^{6}$ in particular.

Detailed studies of the ordered configuration of these fewbody clusters have been performed, mostly by numerical 
simulations, both for systems of vortices in rotating helium and superconducting disks, ${ }^{7,8,31}$ and in quantum dots by a number of authors, notably Peeters and co-workers ${ }^{14,15,32}$ in the classical limit, to whose work we compare our own simulations in Sec. IV below.

\section{Addition spectra}

Addition spectra are built from the energy required to add one extra electron in the trap. This energy is equal to the difference in electrochemical potentials between clusters with $N$ and $N+1$ electrons:

$$
\Delta_{2}(N)=\mu(N+1)-\mu(N)=E(N+1)-2 E(N)+E(N-1),
$$

with $E(N)$ being the ground-state energy of the cluster with $N$ electrons. Once this last quantity has been determined numerically, the values of the experimentally more accessible quantity $\Delta_{2}(N)$ are known. A study of the details of this spectrum thus gives access to the $N$-electron cluster groundstate energies and provides clues about the occurrence of ordering in the Wigner island.

As it became appreciated that addition spectra contain experimentally accessible signatures of the onset for the formation of shells in Wigner molecules, ${ }^{33}$ detailed theoretical predictions for various confinement potentials appeared in the literature (see the reviews by Kouvenhoven et al. ${ }^{34}$ or Reimann and Manninen, ${ }^{20}$ and, for more recent work, notably Ghosal et al. ${ }^{6}$ and Güçlü et al. ${ }^{35}$ ).

In the weakly interacting case, when the last term of the right-hand side of Eq. (1) contributes little, the quantity $\Delta_{2}(N)$ remains small as shells simply fill in and $\mu(N+1)$ $\simeq \mu(N)$. When the addition of an electron triggers the formation of a new shell, for $N=3,6,10,15, \ldots$, the addition energy displays spikes that reveal the shell structure of the $2 \mathrm{D}$ harmonic confinement potential.

As the confinement potential decreases with respect to the Coulomb interaction contribution, i.e., as the electron density $\bar{n}$ diminishes, the Wigner triangular lattice structure eventually forces its imprint on the electron cluster. A new addition energy signature appears with peaks at $N=3,7,11,13, \ldots$ (Ref. 35). The crossover occurs around $n=r_{s}^{-1 / 2} \sim 0.22$ : denser clusters are in a liquidlike state while thinner ones gradually develop a crystal-like state. As emphasized by Ghosal et al. ${ }^{6}$ the transition is smooth; addition spectra are expected to change gradually from shell formations to full spatial ordering. By contrast, the phase diagram in Fig. 1 shows well-marked thresholds for the onset of order as revealed by the study of pair correlations. ${ }^{18}$ It however exhibits the same qualitative trend in the ordering sequence of the electron islands, which can be studied by measuring the addition spectra.

Our goal in the work reported here is a comparison between the addition spectra observed in our experiments and the outcome of numerical simulations that we have performed using the known geometry of the trap. This trap is described in Sec. II, the experimental procedure in Sec. III, the obtained spectra in Sec. IV, and the numerical simulations in Sec. V.

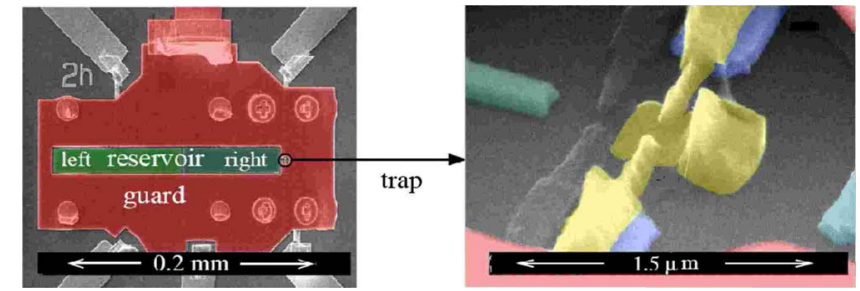

FIG. 2. (Color online) Scanning electron microscopy (SEM) picture of the complete microfabricated device (left) and magnified view of the electron trap (right).

\section{ELECTRON TRAP}

The trap used in the present work to confine electrons to a 2D island is simple compared to other traps used for charged particles, which are usually closed by radio-frequency fields, or for atoms, closed by magneto-optical means. Here, the electron motion is restricted along a plane, on the one side, by the free surface of liquid helium that presents an energy barrier of $\sim 1 \mathrm{eV}$ to the electron and, on the other side, by the image charge in the fluid bulk that provides electrostatic attraction.

At temperatures below $1 \mathrm{~K}$ the saturated vapor pressure of helium is very low: electrons float above the surface of helium in near absolute vacuum. Unlike solids, liquid helium contains no impurities. Its topological defects-the vortices - and its elementary excitations - the phonons, rotons, and ripplons-are well known, allowing relatively simple calculations of interactions between the object in the trap and the environment. ${ }^{36-38}$ A comprehensive review of the physics of electrons on liquid helium can be found in Ref. 39.

The free electron is attracted toward the helium surface by its image charge and is repelled by the $1 \mathrm{eV}$ barrier for entering the liquid bulk; it forms what can be viewed as a one-dimensional hydrogenlike atom. The dielectric constant of helium is low $(\epsilon=1.057)$ and the image charge $Q=e(\epsilon$ $-1) /(\epsilon+1)$, with $e$ being the electron charge, is small. The characteristic length scale along the $z$ axis analogous to the Bohr radius is $a_{B}^{*}=4 a_{B}(\epsilon+1) /(\epsilon-1) \approx 76 \AA$, with $m_{e}$ being the bare electron mass and $a_{B}=0.5292 \AA$ the atomic Bohr radius. The electron in its ground state is floating at $1.5 a_{B}^{*}$ $\approx 114 \AA$ above the helium surface. An electric field can be applied externally to press the electron on the surface, and tune both its height and energy.

If unconfined laterally, the electron moves freely over the surface of bulk helium. Its mobility is the highest of all condensed-matter systems, exceeding $10^{8} \mathrm{~cm}^{2} / \mathrm{V} \mathrm{s}{ }^{40}$ The transverse motion of the electron can be restricted by a system of electrodes located below the helium surface. The microfabricated device used in the present work is shown in Fig. 2 and is described in full detail in Ref. 41.

The right panel of Fig. 2 shows the trap where electrons form a Wigner island. It is a three-dimensional (3D) structure micromachined on a silicon wafer consisting of (i) a ringshaped electrode designed to hold the electrons in a welldefined position over the liquid-helium surface, and (ii) a single-electron transistor (SET) on a pyramidal island at the 


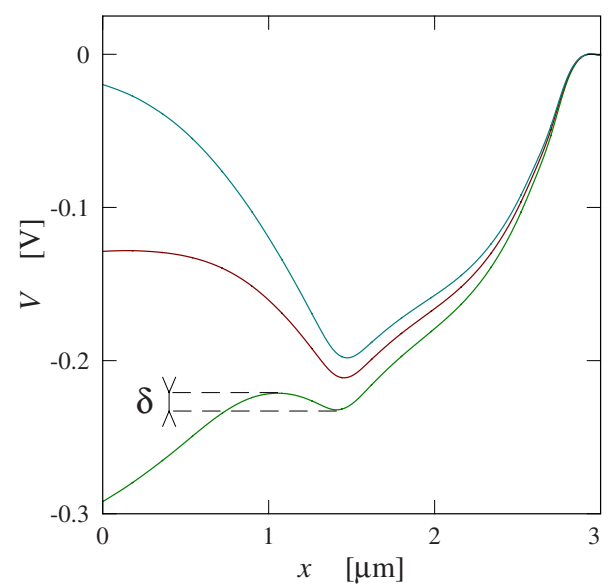

FIG. 3. (Color online) Profile of the electrostatic potential in the plane of symmetry of the trap for $V_{\mathrm{SET}}=0.5,0.2$, and $0 \mathrm{~V}$ from bottom to top. Distances along the $x$ axis are in microns from the edge of the reservoir. The difference between the top of the barrier and the minimum define the confining potential $\delta$. The top of the barrier for $V_{\mathrm{SET}}=0.2$ and $0 \mathrm{~V}$ is in the gorge.

center of the trap used to detect the presence of electronic charges and, possibly, the excitation state of the cluster. The pyramidal structure was built with a five-angle evaporation procedure based on the well-known shadow technique. All electrodes are made of niobium except the SET which is made of aluminum. More details are given in Ref. 42.

The trap and the reservoir, shown in the left panel of Fig. 2 , are $600 \mathrm{~nm}$ lower than the surrounding guard electrode. Both are filled with liquid helium. The long rectangular region acts as a reservoir for surface electron storage. If all electrons in the trap happen to become lost, the trap can be replenished by tapping the reservoir. The guard electrode is made out of a thick $(\sim 0.25 \mu \mathrm{m})$ layer of $\mathrm{Nb}$ deposited on an insulating layer of comparable thickness. This electrode is used to support, by surface tension, the helium film over the reservoir and the trap ring so that the liquid depth is $\sim 0.5 \mu \mathrm{m}$. The electrode itself and the rest of the sample are covered by a thin $(\sim 200-400 \AA)$ film of helium, held by Van der Waals attraction. The bottom of the reservoir is covered by two electrodes, made out of thin niobium. These separate electrodes control the potentials of the right and left halves of the reservoir. They are used to shuffle electrons from one side to the other. The resulting change in capacitance provides a mean of gauging the total mobile charge contained in the reservoir.

A narrow channel across the guard electrodes connects the reservoir to the trap, forming a gorge through which electrons can be forced to move to populate the trap. Under normal operation, the guard electrode is biased to negative potential relative to the SET. A potential barrier thus forms at the gorge, isolating the trap from the reservoir. As the right reservoir electrode protrudes inside the gorge, the potential on this electrode strongly affects the height of this barrier and is preferentially used for tuning. Typical potential profiles along the gorge are shown in Fig. 3. The trap can be made shallow or deep at will by tuning the potentials of the reservoir and the SET island.
The geometric radius of the trap is $3 \mu \mathrm{m}$ but its effective radius is significantly smaller and depends on the configuration of the electrostatic potentials on the various neighboring electrodes. The actual potential well is neither parabolic nor quite axisymmetric. Both shape and depth change with the voltage applied to the electrodes. To set numbers, a trap frequency of $\omega_{0} / 2 \pi$ of $\sim 40 \mathrm{Ghz}$ represents a low estimate when the trap holds few electrons only. The extension of the ground-state wave function of noninteracting electrons is then $l_{0}=\left(\hbar / m_{e} \omega_{0}\right)^{1 / 2} \sim 21.5 \mathrm{~nm}$. The mean distance between interacting electrons $r_{0}$ fixed by the Coulomb energy and the parabolic confining energy is $200 \mathrm{~nm}$, the average density parameter $\bar{n}$ is 0.016 , and the Coulomb energy $E_{C}$ is $83.5 \mathrm{~K}$. At a temperature of $0.2 \mathrm{~K}, \Gamma^{-1}=k_{B} T / E_{C}=0.0024$. Referring to the phase diagram in Fig. 1, the electron island lies well into the radially ordered-phase region, and, moreover, in the near-classical part of that region.

Such electrostatic traps have already been constructed and operated by Glasson et al. ${ }^{43}$ Building on the experience gained in their work, we have improved the design in two ways: (1) the size is made smaller and the electrons are better confined, and (2) the central electrode protrudes from the bottom with a pyramidal shape in order to increase the confinement and the coupling with the electrons (in Fig. 2, right panel), thus enhancing the detection sensitivity.

\section{EXPERIMENTAL PROCEDURE}

\section{A. Electron seeding and monitoring}

We seed electrons by igniting a corona discharge in a small chamber separated from the rest of the cell by a metallic grid with mesh of a few tens of microns. Before the discharge, the cell is heated to a temperature $\sim 1.1 \mathrm{~K}$ at which the vapor pressure becomes high enough. A high vapor pressure is required both to ignite the discharge and to thermalize the electrons so that their energy is lower than the energy barrier of $1 \mathrm{eV}$ needed to penetrate into the liquid. The discharge is ignited by applying $\sim-500 \mathrm{~V}$ to a wire terminated in the middle of the discharge chamber. The typical discharge current is $\sim 0.1-0.2 \mu \mathrm{A}$. The presence of electrons is detected by applying an ac voltage $U_{\text {exc }} \sim 100 \mu \mathrm{V}$ at a frequency of $100 \mathrm{kHz}$ to the right reservoir electrode and monitoring the electromotive force induced on the left electrode with a lock-in amplifier. When electrons appear on the surface, the signal changes by $1-20 \times 10^{-8} \mathrm{~V}$ rms.

After the electrons have been generated and have scattered over the cell, a negative potential is applied to the guard electrode while a positive potential is applied to the reservoir (the potential difference is typically between 0 and $+1 \mathrm{~V}$ during the discharge). These confining potentials localize the electrons primarily over the reservoir and the ringshaped trap.

At low temperature, the electrons over the thin Van der Waals film become localized while electrons over the reservoir and the trap ring, floating much further away from the solid substrate, remain mobile. The thickness of the helium film covering the reservoir and the trap depends somewhat on the amount of helium in the cell. As the level of helium decreases, the film thickness also decreases. When the he- 
lium level drops to $\sim 5 \mathrm{~mm}$ below the sample surface, only a Van der Waals film remains at the center of the reservoir. In a cell with a small volume, it is rather difficult to precisely meter the liquid level as significant amounts of helium can remain trapped in the fill line by surface tension and fountain pressure. To alleviate this problem we have increased the volume of the cell below the sample. We determine the amount required to fill the cell up to the chip level by measuring the capacitance $(\sim 1 \mathrm{pF})$ between two pads on the chip as a function of the volume of helium condensed into the cell. When helium liquid starts covering the chip, the capacitance increases. We then empty the cell and refill it with a quantity of gas corresponding to that just before the capacitance increase.

\section{B. Single-electron transistor readout}

The SET located at the bottom of the trap operates as a sensitive electrometer and quantum amplifier ${ }^{44,45}$ to detect the presence of electrons in the trap and, possibly, their quantum state. This SET is current biased close to the Josephson quasiparticle peak.

We record the Coulomb blockade oscillations obtained by sweeping the reservoir potential. The voltage across the SET, $V_{S}$, is modulated according to the total charge on its island, which reads

$$
q_{I}=\sum_{i} C_{i} U_{i}+\sum_{j} q_{0, j}
$$

The first sum is over all the conductors in the system with capacitance to the island $C_{i}$ and potential $U_{i}$. The second sum runs on the charges induced on the island by free charges in the system, such as charges or dipoles in the substrate and other electrons over helium.

Voltage $V_{S}\left(q_{I}\right)$ across the SET is a periodic function of charge $q_{I}$ with period $e$, the electron charge. However, it also depends on the bias current and the electronic configuration in the cell, and varies from run to run. We determine the functional dependence of $V_{S}\left(q_{I}\right)$ on $q_{I}$ experimentally by sweeping the potential $U_{e}$ of one of the electrodes, usually the one that is swept in subsequent measurements. We then select a portion of the sweep during which the background charge distribution did not change so that several cycles of the function $V\left(q_{I}\right)=V\left(U_{e}\right)$ can be superimposed by transforming $U_{e}$ modulo $P$, where $P$ is an appropriately chosen period. After the modulo transformation the selected piece of data is averaged and interpolated by a smoothing spline function $\operatorname{spl}\left(U_{e}\right)$. The rest of the data is fit piecewise with the function $A \operatorname{spl}\left(U_{e}+q P\right)$, where the amplitude $A$ and the phase $q$ are fitting parameters. The amplitude is also taken as a free fitting parameter because the observed peak amplitude $V\left(U_{e}\right)$ varies slightly with $U_{e}$.

The phase $q$ is the charge, expressed as a fraction of $e$, induced on the SET island by free charges in the system. The expected value can be estimated using the reciprocity principle: it is equal to that induced at the location of an electron when the SET island is biased with unit potential and all the other conductors in the system are grounded. We have calculated this potential numerically, using the actual 3D geom-

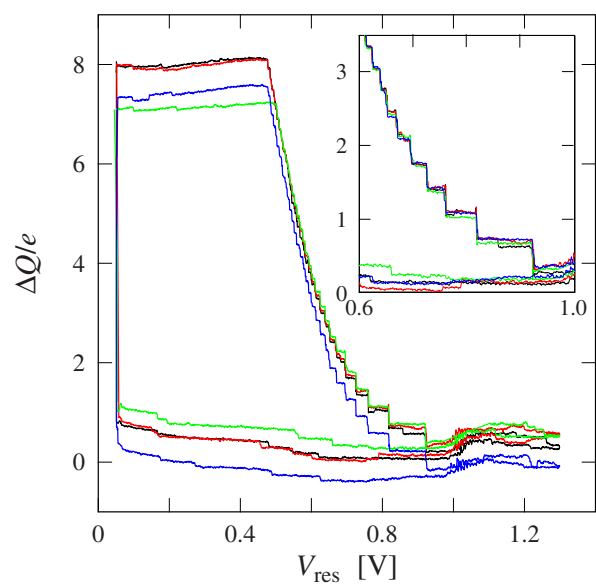

FIG. 4. (Color online) Reduced charge on the SET island in terms of the potential of the reservoir electrode for several sweeps of $V_{\text {res. }}$. The SET potential is $+0.6 \mathrm{~V}$ while the guard electrode potential is 0 . The inset shows the steps of the staircase for low occupation numbers down to zero, corrected for the baseline drift occurring between the different sweeps of $V_{\text {res. }}$.

etry of the trap inferred from SEM photographs, to obtain a value for the charge $q$ of $0.5 e$.

To reduce the noise due to fluctuations of the dc voltage across the SET, we apply a low-frequency $(80-150 \mathrm{~Hz})$ modulation with amplitude $\sim 100 \mu \mathrm{V}$ to the guard electrode. The amplitude of the detected signal is proportional to the derivative of the periodic function $V_{S}\left(q_{I}\right)$.

\section{Trapping electrons}

After electrons are seeded, we let the system cool down from $1 \mathrm{~K}$ and proceed to load the trap with a given number of them. Typically, at this stage, the guard electrode is biased to a negative potential, between -0.1 and $-0.5 \mathrm{~V}$, and the SET is biased to a positive potential between 0 and $0.5 \mathrm{~V}$. First, we fill the trap with electrons by lowering the voltage applied to the reservoir electrodes. The electrons are repelled toward the trap. The values of the electrode potentials are determined by trial and error. Usually, at least one of the reservoir electrodes stays positive with respect to the guard although we found that we can keep the electrons even with both electrodes more negative than the guard. Often, the charging potentials have to be lowered in the course of the experiment as the electron reservoir gradually gets depleted.

After the trap is charged, we start sweeping the potential of the right reservoir electrode $V_{\text {res }}$. When $V_{\text {res }}$ is low, the barrier is high and the ring remains full of electrons. Sweeping up this voltage reduces the barrier height. When the barrier becomes low enough, electrons start leaving the ring. This escape suddenly changes the charge on the SET island. The SET island charge variation manifests itself as a phase jump in the Coulomb blockade oscillations (see Fig. 5, in which the arrow points to the phase jump). Referring to the traces in Fig. 4, the SET detects a sudden change in the charge at $V_{\text {res }} \sim 0.48 \mathrm{~V}$. Since the SET measures charge modulo $e$, we cannot determine directly the absolute value of the total charge but we know that the trap flooded with electrons has started to empty. 


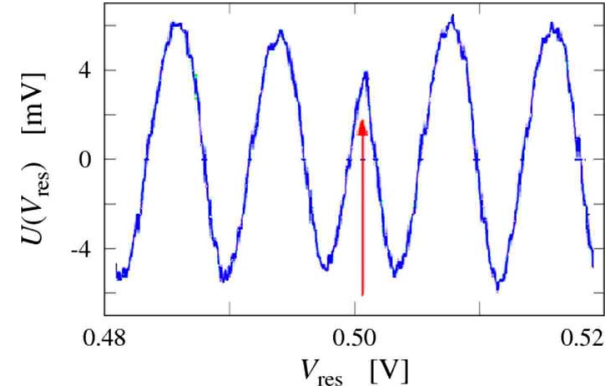

FIG. 5. (Color online) Variation in the SET output signal $U$ when the reservoir voltage, $V_{\text {res }}$, is swept. The Coulomb blockade oscillation is interrupted by a change of the charge in the SET island at $\sim 0.5 \mathrm{~V}$ as marked by the arrow.

In this initial phase, electrons leave the trap in such a way that individual escapes cannot be resolved. But, as $V_{\text {res }}$ is raised further and the barrier height decreases, clear steplike jumps become visible. Each electron leaving the trap changes the induced charge on the SET island. The corresponding jump of the SET island charge, $\Delta Q$, is plotted in Fig. 4 against $V_{\text {res }}$. Its amplitude depends on the number of the electrons left in the trap and amounts to $\sim 0.4 e$ when few electrons only are left. This value compares well with that obtained from finite-element calculations of the electrostatic field using the known trap geometry, $\sim 0.5 e$ : this good agreement confirms that electrons escape from the trap one at a time. At $0.93 \mathrm{~V}$ in Fig. 4 the last electron leaves the trap. Between 0.82 and $0.93 \mathrm{~V}$, only one electron is left in the trap. The previous step to the left corresponds to two electrons in the trap, and so on.

The length of these steps reflects the change in electrochemical potential that leads to the removal (escape) of one electron. As shown in the inset, this quantity is well determined experimentally. The variation in $V_{\text {res }}$ between the jumps depends on the Coulomb repulsion between the electrons. Stair length and height increase when the number of electrons in the trap decrease. Length increase means a larger reduction in the barrier to extract one electron (electronelectron interactions decrease). Height increase means that the leaving electron induces a larger charge on the island, i.e., the leaving electron is closer to the center of the island.

These results are similar to the results obtained at the Royal Holloway in London. ${ }^{43}$ The main difference lies in significantly better defined steps due to improved coupling with the pyramidal SET and to the smaller size of the trap, leading to stronger repulsion between the electrons. The positions of the steps are also more stable in our experiments, as seen in the inset of Fig. 4, and amenable to precise quantitative analysis.

\section{EXPERIMENTAL ADDITION SPECTRA}

In the following, we shift our attention from SET-phase variation signals to addition spectra, which are inherently more reproducible for the following reason. For a given set of external parameter values, such as the potentials applied to different electrodes, the potential for which one electron

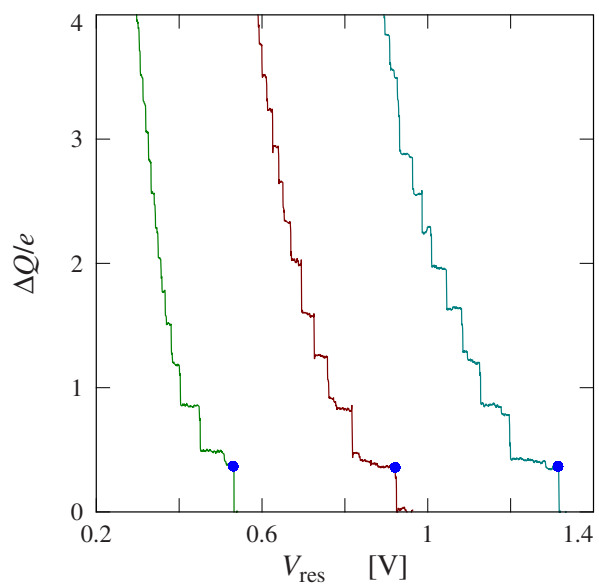

FIG. 6. (Color online) SET-phase variation for the values of the potential on the SET, $V_{\mathrm{SET}}$, of $0.3,0.5$, and $0.8 \mathrm{~V}$ from left to right. The dots mark the escape of the last electron from the trap.

leaves the trap can be shifted due to modifications in the distribution of trapped charges in the substrate. This spurious effect is more likely to occur shortly after a new corona discharge, which requires a "high" temperature (1-1.2 K) and involves a high voltage. As addition spectra are the difference between $V_{\text {res }}(N-1)$ and $V_{\text {res }}(N)$, namely, $\Delta \mu(N)$ $=V_{\text {res }}(N)-V_{\text {res }}(N-1)$, potential drifts are removed.

The contact potential between niobium and aluminum in the cell modifies the applied value of $V_{\text {res }}$ and has to be accounted for. This potential is determined in the experimental setup as follows. We read in Fig. 6 the value of the reservoir potential for which the last electron leaves the trap for different potentials applied to the SET (i.e., different sizes of the trap). These values are plotted against $V_{\mathrm{SET}}$ in Fig. 7. The experimental points fall on a straight line that cuts the $x$ axis for $V_{\text {res }}=-0.206 \pm 0.005 \mathrm{~V}$. This value represents, to a weak correction due to the image charge of the remaining electron, the contact potential between niobium and aluminum. It must be taken into account to obtain the true value of the potential that acts on the electrons.

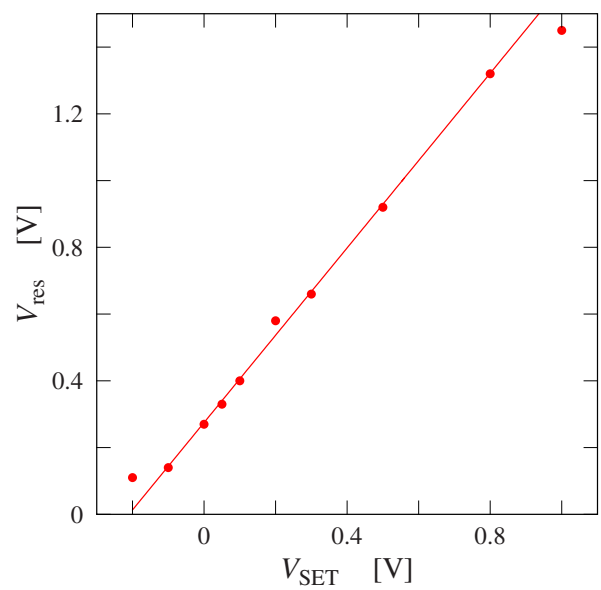

FIG. 7. (Color online) Potential of the reservoir, $V_{\text {res }}$, at which the last electron leaves the trap. The intercept with the $x$ axis yields the effective contact potential between aluminum and niobium, found to be $0.206 \pm 0.005 \mathrm{~V}$. 


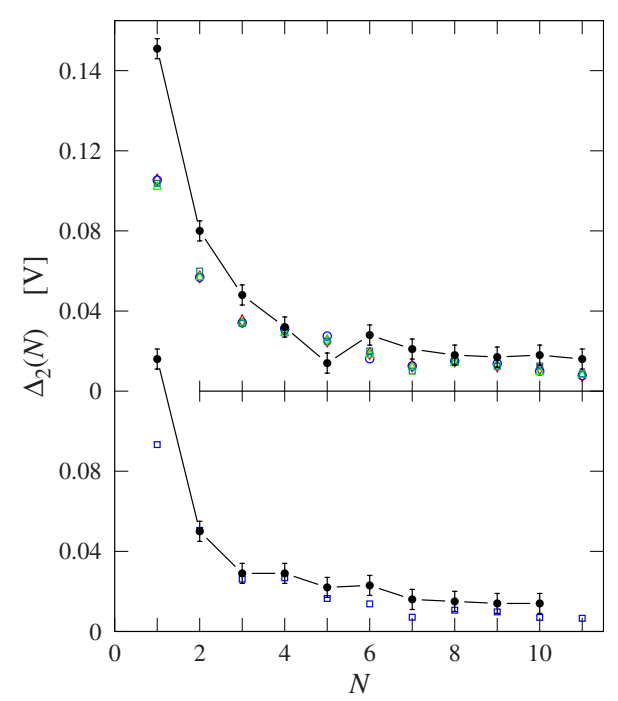

FIG. 8. (Color online) Top: addition spectrum for $V_{\mathrm{SET}}=0.5 \mathrm{~V}$ $\left(V_{\mathrm{SET}}=0.706 \mathrm{~V}\right.$ in the simulations). Squares, $(\square)$, lozenges, $(\diamond)$, triangles, $(\triangle)$, and circles, $(\bigcirc)$, are experimental data for different runs and fall nearly on top of one another. Bottom: addition spectrum for $V_{\mathrm{SET}}=0.3 \mathrm{~V}\left(V_{\mathrm{SET}}=0.506 \mathrm{~V}\right.$ in the simulations $)$; squares are experimental results. Dots, $(\mathbf{O})$, are the results of the MC simulations, linked by straight-line segments to guide the eye. Error bars on the simulated data represent an estimate of the uncertainty.

The addition spectra themselves are obtained by plotting the stair length of staircaselike patterns, such as shown in Fig. 6, in terms of the number of electrons in the island. Experimentally observed addition spectra are given in Fig. 8 for two trap sizes corresponding to $V_{\mathrm{SET}}=0.5$ and $0.3 \mathrm{~V}$, respectively. Different potential sweeps following a given corona discharge are shown on the same graph in Fig. 8 (top panel): the experiment gives quite reproducible results.

\section{MONTE CARLO SIMULATIONS}

In order to interpret the details of these experimental results, we have carried out Monte Carlo simulations of the addition spectra that correspond to the precise shape of the confining potential well in the experimental trap. These MC simulations are based on the procedure described by $\mathrm{Be}$ danov and Peeters. ${ }^{14}$ In the low-density limit, which is the regime attained here, the electrons occupy only a small fraction of the total area of the trap. They are (mostly) distinguishable and can be treated as classical particles. This simplification is also made by Peeters and co-workers. ${ }^{14,15,32}$

Our trap is not parabolic but its profile can be determined by finite element calculation based on its known geometry (see Fig. 3). In doing so, we have attempted to reproduce as accurately as possible the pyramidal shape of the SET island. The values of the confining potential profile have been computed for a finite number of nodes and interpolated with parabolas to arbitrary coordinates $\mathbf{r}_{i}$.

In order to check our computational procedure, we have reproduced Bedanov and Peeters' ${ }^{\prime 4}$ results for a parabolic trap defined analytically. We discretized the parabolic potential with the same number of nodes as for the actual potential

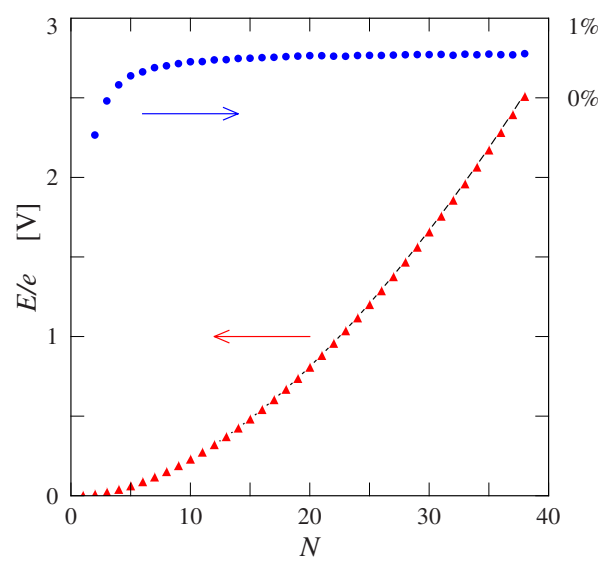

FIG. 9. (Color online) Increase in the free energy of the cluster with electron number in a parabolic trap, in volt: $(\mathbf{\Lambda})$, our results based on a discrete parabolic profile; solid line, Kong et al.'s results (Ref. 46). The relative difference in percent between the two calculations is plotted at the top, $(\mathbf{)})$, referred to the vertical scale on the right.

determination for our cell. We then performed the same MC simulations to find the energy and the configuration of a known number of electrons in the trap. Our results, shown in Fig. 9, fall very well in line with the published results, those of Ref. 14 and the more recent results of Kong et al. ${ }^{46}$ The small systematic difference of $\sim 0.75 \%$ seen in Fig. 9 might possibly be due to the discretization of the confinement potential. Our simulations of the addition spectra are also in very good agreement with those of Peeters and co-workers, ${ }^{14,15,32}$ as shown in Fig. 10.

Finally, we compare electronic configurations. The ground state of the configuration with 31 electrons is one of the most difficult to find due to the closeness of the first metastable state (the difference is only $0.004 \%$ ). The result, shown in Fig. 11, is once again in agreement with Peeters and co-workers. ${ }^{14,15,32}$ We thus are quite confident that our MC simulations lead to the true ground-state configurations and to a precise evaluation of their energies.

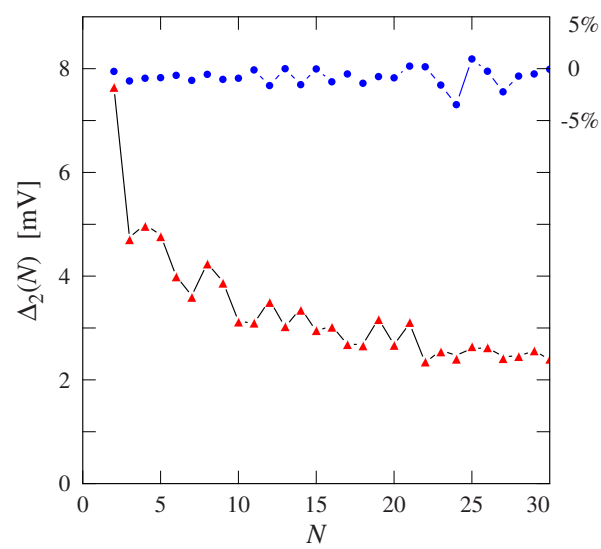

FIG. 10. (Color online) Computed addition spectra in this work and that of Bedanov and Peeters (Ref. 14), confirmed by Kong et al. (Ref. 46), ( $\mathbf{\Delta})$, at the bottom. The relative difference in percent between these calculations is plotted at the top, $(\mathbf{O})$, referred to the vertical scale on the right. 


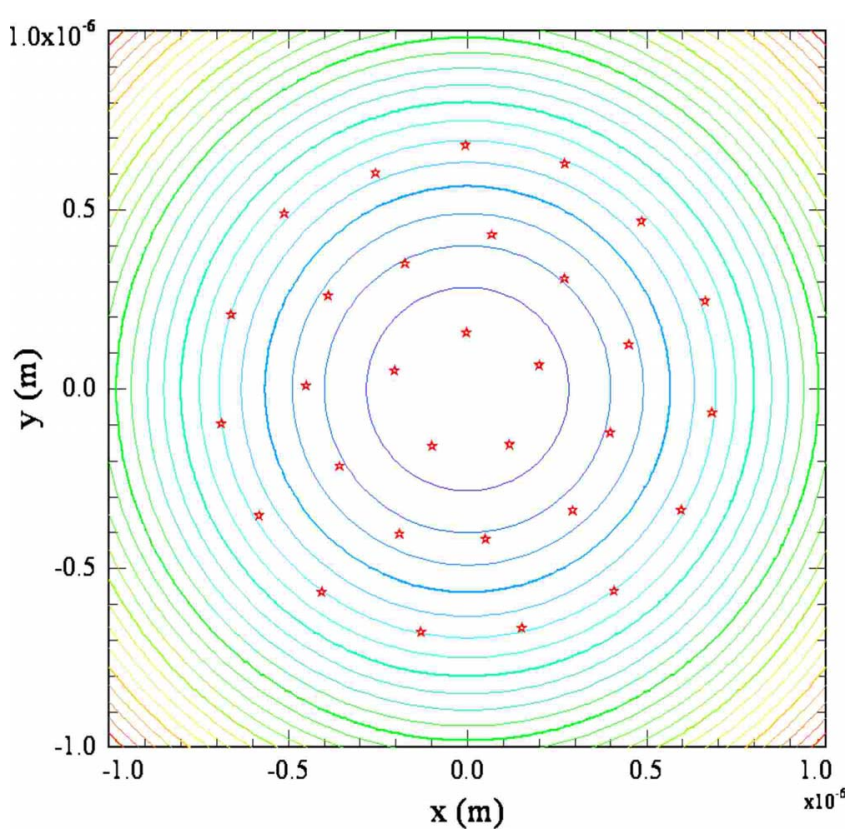

FIG. 11. (Color online) Wigner molecule in a parabolic trap with 31 electrons. Lines represent potential contours; the minimum of the parabola is centered on $(0,0)$. Stars represent the electrons. The ground-state configuration is the configuration $(5,11,15)$ as in Kong et al. (Ref. 46).

We now turn to the case of addition spectra in the actual (nonparabolic) trapping potential, for which we need the electron energy. This energy has two parts: an electrostatic part and the repulsion due to the Coulomb interaction. The electrostatic part can be attractive if electrons are in the trap or repulsive if the electrons are on the other side of the barrier (see Fig. 3). The electron energy reads in the classical limit, discarding the kinetic-energy term in Eq. (1):

$$
E\left(N, V_{\text {res }}\right)=-\sum_{i=1}^{N} e V\left(\mathbf{r}_{i}\right)+\frac{e^{2}}{4 \pi \epsilon_{0}} \sum_{j<i}^{N} \frac{1}{\left|\mathbf{r}_{i}-\mathbf{r}_{j}\right|} .
$$

The electrostatic energy where $e V\left(\mathbf{r}_{i}\right)$ at $\mathbf{r}_{i}$, which is the $i$ th electron location, is that obtained by the finite element calculation. The second term in Eq. (4) represents the Coulomb interaction energy between electrons, which is only around $10 \%$ of the total energy; the bulk part of the energy comes from the trapping potential.

An electron leaves the trap when its energy overcomes the confining energy barrier $\delta$, which is the energy difference between the minimum and the energy at the barrier (see Fig. 3 ). That is, a given configuration is stable as long as the average energy per electron remains lower than $\delta$ : $E_{N}$ $=E\left(N, V_{\text {res }}\right) / N \leq \delta$. Here, it is assumed that the electrons in the island have identical energies.

We first compute the evolution of the confining potential $\delta$ in terms of the potential applied to the reservoir. The contact potential must be taken into account: when the potentials applied to the SET electrode and to the reservoir electrode are $V_{\mathrm{SET}}$ and $V_{\text {res }}$, the potentials used in the simulations are $V_{\mathrm{SET}}+0.206 \mathrm{~V}$ on the SET electrode and $V_{\text {res }}$ is left unchanged on the reservoir electrode. For $N$ electrons in the

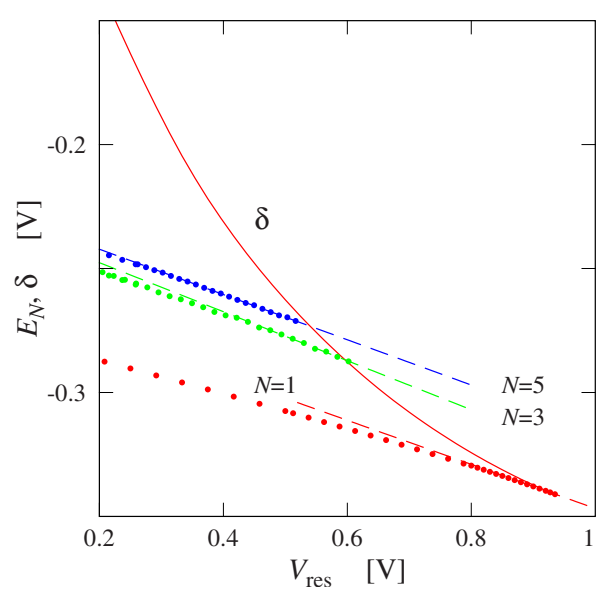

FIG. 12. (Color online) Solid line: confining potential $\delta$ in terms of the potential applied on the reservoir electrode $V_{\text {res }}$. Dots are Monte Carlo simulations of the average energy per electron for one, three, and five electrons in the trap. Dash-dash lines are linear extrapolations of the Monte Carlo results as explained in the text. The crossing point between $\delta$ and this average energy gives the potential for which an electron leaves the trap.

trap, we compute the total energy for different values of the reservoir potential. When the barrier is too low-i.e., when the reservoir potential is too close to the threshold when the electron is about to escape-the Monte Carlo simulations fail to find the ground state. The starting temperature in the simulations is too high and allows electrons to escape readily over the barrier. Using a lower starting temperature to circumvent the problem does not lead to the real ground state of the configuration.

To resolve this issue we calculated the energy for values of the reservoir potential slightly lower than the escape threshold, for which the simulations did find the ground state, and extrapolated the results linearly to higher values of this potential. This procedure is illustrated in Fig. 12, which shows the evolution of the confining potential and the average energy per electron for $N=1,3$, and 5 in terms of the reservoir voltage. An electron leaves the trap when these curves intersect.

The comparison between the outcome of these calculations and the experimental results for, respectively, $V_{\mathrm{SET}}$ $=0.5$ and $0.3 \mathrm{~V}$ is shown in Fig. 8. The size and the shape of the trap depend on $V_{\text {SET }}$ and the results are significantly different. The scatter on the experimental points is less than the uncertainty on the simulations. The observed addition spectra are quite reproducible as long as the cell is kept cold, below $1 \mathrm{~K}$. When it becomes necessary to replenish the electron reservoir, the temperature is raised and a new corona discharge is ignited. Then the distribution of stray charges changes and the addition spectra fine structure, which is quite sensitive to the potential profile, also changes. The differences between the calculated and observed spectra may be in part due to this uncontrolled influence of random surface charges.

A recurrent feature of the observed spectra is a peak for $N=6$ followed by a trough for $N=7$. Referring to the work of Güçlü et al., ${ }^{35}$ this indicates that electrons order in shells and not on a triangular lattice, which would give a peak at $N$ 
$=7$. The corresponding trap frequency is $\sim 60 \mathrm{GHz}$ and the electronic density $\bar{n} \simeq 0.017$. These observations fit well into the phase diagram shown in Fig. 1 according to which, at an electron temperature of $\sim 200 \mathrm{mK}$, the cluster is radially oriented (formation of shells) but not orientationally oriented.

For very few electrons $(N \leq 3)$, the Monte Carlo simulation yields a significantly larger addition energy than what is observed. Even for $N>3$, the calculated addition energies usually cap the experimental ones. This observation probably means that the Wigner island lies in an excited configuration. Its energy is higher than that of the ground state; a lesser decrease in the barrier is required to extract one electron. On the contrary, higher values than the calculated ones remain unexplained (e.g., $N=5$ for the top panel in Fig. 8). The extraneous source of noise energy that seems to be present in the experiment possibly comes from the tight coupling with the SET. We have evidence that the SET back action affects the temperature of the electrons in the island in a way that depends on the SET bias current, either heating or cooling with respect to the bath temperature. This effect is under study.

It is known from the moving pictures of vortex line configuration in a superfluid rotating bucket by Williams and Packard $^{23}$ that the vortices tend to jump around randomly. In order to damp the vortex motions so that they could be photographed, the authors of Ref. 23 added ${ }^{3} \mathrm{He}$ impurities to the ${ }^{4} \mathrm{He}$ superfluid to bring in some dissipation. In our Wigner islands, no such dampener is introduced. Due to the extremely high mobility of electrons over helium, it is quite likely that the electronic configuration is also extremely unsteady.

\section{CONCLUSION}

We have studied the confinement of a small number of electrons in a trap over a liquid-helium film. Stable configurations down to a single electron can be obtained reproducibly and their energy recorded with an SET readout. The experimental addition spectra compare well with those obtained in Monte Carlo simulations in a trapping potential directly derived from the cell geometry. Charges trapped in the dielectric parts of the sample only weakly modify the potential profile. The good agreement between actual experiments and MC simulations (1) confirms the validity of the model (and assumptions) used in the simulation, (2) shows that no uncontrolled, or unforeseen, feature plays a significant role in the physical system, and (3) opens the way, once the problem of repeatability of addition spectra after corona discharges is solved, to more detailed studies of these Wigner islands, and, in particular, of orientational ordering.

\section{ACKNOWLEDGMENTS}

This work was supported in part by Fonds National de la Science Grant No. ACI 2002-2140. It was started as a collaboration with the Royal Holloway College in the framework of the European Network Contract No. HPRN-CT200000157.
*Present address: Laboratoire EM2C-CNRS-École Centrale Paris 92295 Châtenay-Malabry, France; em.rousseau@gmail.com

†Present address: Chemistry Dept., North Carolina State Univ., Raleigh, NC 27695, USA.

${ }^{\ddagger}$ Present address: Science Faculty, American Community Schools of Athens, 129 Aghias Paraskevis, 15234 Halandri, Athens, Greece. ${ }^{1}$ E. Wigner, Phys. Rev. 46, 1002 (1934).

${ }^{2}$ C. C. Grimes and G. Adams, Phys. Rev. Lett. 42, 795 (1979).

${ }^{3}$ V. Shikin, Sov. Phys. Usp. 32, 452 (1989).

${ }^{4}$ B. Tanatar and D. M. Ceperley, Phys. Rev. B 39, 5005 (1989).

${ }^{5}$ X. Waintal, Phys. Rev. B 73, 075417 (2006).

${ }^{6}$ A. Ghosal, A. D. Güçlü, C. J. Umrigar, D. Ullmo, and H. U. Baranger, Phys. Rev. B 76, 085341 (2007).

${ }^{7}$ D. Stauffer and A. Fetter, Phys. Rev. 168, 156 (1968).

${ }^{8}$ L. J. Campbell and R. M. Ziff, Phys. Rev. B 20, 1886 (1979).

${ }^{9}$ A. Abrikosov, Sov. Phys. JETP 5, 1174 (1957).

${ }^{10}$ D. Cribier, B. Jacrot, L. M. Rao, and B. Farnoux, Phys. Lett. 9, 106 (1964).

${ }^{11}$ U. Essmann and H. Trauble, Phys. Lett. 24A, 526 (1967).

${ }^{12}$ Y. E. Lozovik and V. I. Yudson, JETP Lett. 22, 11 (1975).

${ }^{13}$ M. Shayegan, in Topological Aspects of Low Dimensional Systems, edited by A. Comtet, T. Jolicoeur, S. Ouvry, and F. David (Springer-Verlag, Berlin, 1999), p. 1, Les Houches Summer School Session LXIX.

${ }^{14}$ V. M. Bedanov and F. M. Peeters, Phys. Rev. B 49, 2667 (1994).

${ }^{15}$ V. A. Schweigert and F. M. Peeters, Phys. Rev. B 51, 7700
(1995).

${ }^{16}$ A. A. Koulakov and B. I. Shklovskii, Phys. Rev. B 57, 2352 (1998).

${ }^{17}$ R. Egger, W. Häusler, C. H. Mak, and H. Grabert, Phys. Rev. Lett. 82, 3320 (1999).

${ }^{18}$ A. V. Filinov, M. Bonitz, and Y. E. Lozovik, Phys. Rev. Lett. 86, 3851 (2001); M. Bonitz et al., Phys. Plasmas 15, 055704 (2008).

${ }^{19}$ A. Harju, S. Siljamäki, and R. M. Nieminen, Phys. Rev. B 65, 075309 (2002).

${ }^{20}$ S. M. Reimann and M. Manninen, Rev. Mod. Phys. 74, 1283 (2002).

${ }^{21}$ M. Saint Jean, C. Even, and C. Guthmann, Europhys. Lett. 55, 45 (2001).

${ }^{22}$ I. V. Grigorieva, W. Escoffier, J. Richardson, L. Y. Vinnikov, S. Dubonos, and V. Oboznov, Phys. Rev. Lett. 96, 077005 (2006).

${ }^{23}$ G. A. Williams and R. E. Packard, Phys. Rev. Lett. 33, 280 (1974).

${ }^{24}$ U. Parts, M. H. Ruutu, H. Koiwniemi, Yu. M. Bunkov, V. Dmitriev, V. M. Fogelström, M. Huebner, Y. Kondo, N. B. Kopnin, J. S. Korhonen, M. Krusius, O. V. Lounasmaa, I. Soininen, and G. E. Volovik, Europhys. Lett. 31, 449 (1995).

${ }^{25}$ G. Papageorgiou , P. Glasson, K. Harrabi, V. Antonov, E. Collin, P. Fozooni, P. G. Frayne, M. J. Lea, D. G. Rees, and Y. Mukharsky, Appl. Phys. Lett. 86, 153106 (2005).

${ }^{26}$ D. Pfannkuche, V. Gudmundsson, and P. A. Maksym, Phys. Rev. 
B 47, 2244 (1993).

${ }^{27}$ E. Anisimovas and A. Matulis, J. Phys.: Condens. Matter 10, 601 (1998)

${ }^{28}$ K. Balzer, C. Nölle, M. Bonitz, and A. Filinov, J. Phys.: Conf. Ser. 35, 209 (2006).

${ }^{29}$ N. Simonović, Few-Body Syst. 38, 139 (2006).

${ }^{30}$ Considerably lower critical values for $r_{s}$, down to four, corresponding to much higher critical densities, have been reported for clusters with few electrons (Ref. 17). However, these findings seem to be an artifact of the computational scheme and are not confirmed in subsequent works (Refs. 6, 47, and 48). Thus, the critical density beyond which quantum fluctuations dominate, destroying the ordered phase of $2 \mathrm{D}$ confined structures, remains of the same order of magnitude as for the opengeometry situation when the number of particles, $N$, is large, as pictured in Fig. 1.

${ }^{31}$ V. A. Schweigert, F. M. Peeters, and P. S. Deo, Phys. Rev. Lett. 81, 2783 (1998).

${ }^{32}$ B. Szafran, F. M. Peeters, S. Bednarek, and J. Adamowski, Phys. Rev. B 69, 125344 (2004).

${ }^{33}$ S. Tarucha, D. G. Austing, T. Honda, R. J. van der Hage, and L. P. Kouwenhoven, Phys. Rev. Lett. 77, 3613 (1996).

${ }^{34}$ L. Kouwenhoven, D. Austing, and S. Tarucha, Rep. Prog. Phys. 64, 701 (2001).

${ }^{35}$ A. D. Güçlü, A. Ghosal, C. J. Umrigar, and H. U. Baranger, Phys. Rev. B 77, 041301(R) (2008).

${ }^{36}$ D. S. Fisher, B. I. Halperin, and P. M. Platzman, Phys. Rev. Lett.
42, 798 (1979).

${ }^{37}$ M. I. Dykman, P. M. Platzman, and P. Seddighrad, Phys. Rev. B 67, 155402 (2003).

${ }^{38}$ M. Lea, P. Frayne, and Y. Mukharsky, Fortschr. Phys. 48, 1109 (2000).

${ }^{39}$ Two Dimensional Electron Systems on Helium and Other Cryogenic Substrates, edited by E. Y. Andrei (Kluwer Academic, Dodrecht, 1997).

${ }^{40}$ K. Shirahama and K. Kono, Phys. Rev. Lett. 74, 781 (1995); K. Shirahama, S. Ito, H. Suto, and K. Kono, J. Low Temp. Phys. 101, 439 (1995).

${ }^{41}$ E. Rousseau, D. Ponarine, E. Varoquaux, and Y. Muhkarsky, J. Low Temp. Phys. 148, 193 (2007).

${ }^{42}$ E. Rousseau, Ph.D. thesis, Université Paris XI (2006); http:// tel.archives-ouvertes.fr/tel-00250371/fr/.

${ }^{43}$ P. Glasson, G. Papageorgiou, K. Harrabi, V. Antonov, E. Collin, P. Fozooni, P. G. Frayne, M. J. Lea, Y. Mukharsky, and D. G. Rees, J. Phys. Chem. Solids 66, 1539 (2005).

${ }^{44}$ M. Devoret and R. Schoelkopf, Nature (London) 406, 1039 (2000).

${ }^{45}$ K. Likharev, Nano et Micro Technologies 3, 71 (2003).

${ }^{46}$ M. Kong, B. Partoens, and F. M. Peeters, Phys. Rev. E 65, 046602 (2002).

${ }^{47}$ See S. M., Reimann, M. Koskinen, and M. Manninen, Phys. Rev. B 62, 8108 (2000), and references therein.

${ }^{48}$ B. Reusch, W. Häusler, and H. Grabert, Phys. Rev. B 63, 113313 (2001). 